\section{On the Use of SDA for the Analysis of Boxed Planar Lines with Complex Media}

\author{
Gonzalo Plaza, Francisco Mesa, and Francisco Medina
}

\begin{abstract}
This paper discusses the conditions under which the spectral-domain approach (SDA) can be applied to the analysis of boxed planar lines when complex materials (anisotropic dielectrics, ferrites, magnetoplasmons, chiral media, and so on) are used as substrates. It will be shown that whereas SDA can always be efficiently applied to study laterally open structures, the simultaneous presence of lateral boundary conditions and nonisotropic materials requires further study. Thus, the symmetry properties of the constitutive dyadics that makes possible a rigorous application of the SDA to those kinds of structures will be reported in this paper.
\end{abstract}

Index Terms-(Bi)anisotropic media, shielded planar lines, spectral domain.

\section{INTRODUCTION}

The technological advances in the development of complex materials has raised a theoretical and practical interest in the analysis of microwave transmission lines and devices with substrate layers of very general properties. This research topic is expected to lead to new microwave devices and/or to enhance the performance of the currently existing ones. In this way, a great effort is also devoted to study the propagation and/or radiation characteristics of a number of planar structures whose layers are made of anisotropic, gyrotropic, or bi(an)isotropic materials. Among the most common techniques to deal with planar structures, those based on the application of Fourier transform (FT) techniques, such as the spectral-domain approach (SDA), have proven to be very efficient [1]. This method has been successfully extended to deal with anisotropic and bi(an)isotropic substrates in laterally open structures [2], [3]. Laterally shielded configurations having certain particular anisotropic media have also been properly treated in the literature [4]-[6]. However, the direct application of the SDA expressions (originally developed for laterally open structures) to the analysis of boxed structures needs a careful examination when nonisotropic materials are involved [7]-[10]. The inappropriate use of the SDA to study, for example, boxed guiding structures containing longitudinally magnetized ferrite/semiconductor and/or chiral materials, could bring conceptual errors that would yield inaccurate numerical results. Thus, the presence of lateral electric walls (EWs) and/or magnetic walls (MWs) requires deeper examination. Although this point has been addressed in the literature for certain specific cases in various contexts [11]-[14], it seems that some practitioners of the SDA are not completely aware of this fact, perhaps because a comprehensive discussion on this topic in the frame of the SDA has not yet been reported. Thus, the main goal of this paper is to clarify the conditions under which the SDA is suitable for the analysis of boxed planar lines including arbitrary complex linear media as substrates. After addressing the nature of the problem with simple examples, the study will provide criteria (related to the symmetry properties of the constitutive parameters of the materials) to know when the SDA is feasible to analyze the above-mentioned type of guiding structures.

Manuscript received November 10, 1999; revised September 27, 2000. This work was supported by the Spanish Comision Interministerial de Ciencia y Tecnologia under Project TIC98-0630.

G. Plaza and F. Mesa are with the Department of Applied Physics I, University of Seville, 41012 Seville Spain (e-mail: mesa@cica.es).

F. Medina is with the Department of Electronics and Electromagnetism,

School of Physics, University of Seville, 41012 Seville Spain.

Publisher Item Identifier S 0018-9480(01)05046-3.

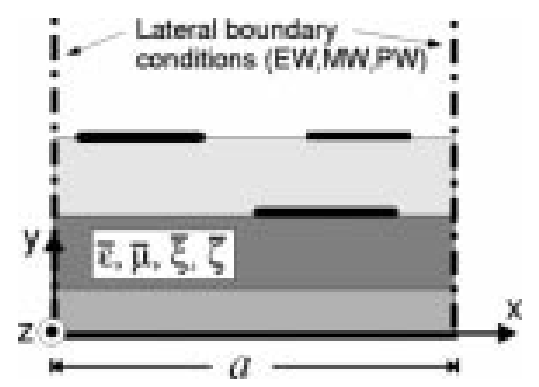

Fig. 1. Cross section of a planar layered line. The constitutive dyadics of each layer are assumed to be homogeneous for $0<x<a$.

\section{APPLICATION OF SDA to PlanAR LiNES}

Although the SDA is actually a well-known and widely used technique, it will be briefly outlined here to survey the possible drawbacks appearing when the technique is applied to planar lines with lateral boundary conditions (BC) and nonisotropic materials. Fig. 1 shows the cross section of a generic layered planar line with rectangular BCs: EWs, MWs, or periodic walls (PWs) exist at $x=0$ and $x=a$. (The absence of these walls leads to laterally open lines.) In general, the substrate layers are homogeneous linear materials whose constitutive parameters are given by the following linear dyadics:

$$
\begin{aligned}
\mathbf{D} & =\overline{\boldsymbol{\epsilon}} \cdot \mathbf{E}+\overline{\boldsymbol{\xi}} \cdot \mathbf{H} \\
\mathbf{B} & =\bar{\zeta} \cdot \mathbf{E}+\overline{\boldsymbol{\mu}} \cdot \mathbf{H} \\
{\left[\begin{array}{l}
\mathbf{D} \\
\mathbf{B}
\end{array}\right] } & =[\mathbf{\Gamma}] \cdot\left[\begin{array}{l}
\mathbf{E} \\
\mathbf{H}
\end{array}\right]
\end{aligned}
$$

which account for the simplest isotropic dielectric to the most complex bianisotropic material.

Assuming a field dependence of the type $\mathbf{A}(\mathbf{r}, t)=$ $\mathbf{A}(x, y) \exp \left[-j\left(k_{z} z-\omega t\right)\right]$, Maxwell curl equations can be written as

$$
\left[\begin{array}{cc}
-\overline{\mathcal{R}} & \mathbf{0} \\
\mathbf{0} & \overline{\mathcal{R}}
\end{array}\right] \cdot\left[\begin{array}{l}
\mathbf{E} \\
\mathbf{H}
\end{array}\right]=j \omega\left[\begin{array}{l}
\mathbf{B} \\
\mathbf{D}
\end{array}\right]=j \omega\left[\begin{array}{cc}
\bar{\zeta} & \overline{\boldsymbol{\mu}} \\
\overline{\boldsymbol{\epsilon}} & \bar{\xi}
\end{array}\right] \cdot\left[\begin{array}{c}
\mathbf{E} \\
\mathbf{H}
\end{array}\right]
$$

where $\overline{\mathcal{R}}=(\hat{\mathbf{x}} \hat{\mathbf{y}}-\hat{\mathbf{y}} \hat{\mathbf{x}}) j k_{z}+(\hat{\mathbf{y}} \hat{\mathbf{z}}-\hat{\mathbf{z}} \hat{\mathbf{y}}) \partial / \partial x+(\hat{\mathbf{x}} \hat{\mathbf{z}}-\hat{\mathbf{z}} \hat{\mathbf{x}}) \partial / \partial y$ is the curl operator. Eliminating now the $y$-components of the fields, the following matrix first-order partial differential equation [subject to the appropriate BCs $(x, y)$ ] can be formally found for $\mathbf{X}=\left[E_{x}, E_{z}, H_{x}, H_{y}\right][2]$ :

$$
\left.\begin{array}{c}
{\left[\mathcal{D}\left(\partial / \partial x, \partial / \partial y, k_{z}, \omega\right)\right][\mathbf{X}]=[\mathbf{Q}(\omega)][\mathbf{X}]} \\
\operatorname{BC's}(x, y)
\end{array}\right\}
$$

where $[\mathcal{D}(\cdot)]$ is the resultant first-order matrix differential operator and $[\mathbf{Q}(\omega)]$ is a matrix accounting for the layered medium. The standard application of the SDA [1] to solve for the above partial differential equation consists basically of applying a combination of Green's function methods and an appropriate spectral representation.

Next, let us examine the different BCs in the lateral $x$-direction in connection with the application of FTs.

\section{A. Laterally Open Lines}

This case shows an open domain in the lateral direction since there are not BCs in the $x$-direction. Functions of the type $\exp \left(-j k_{x} x\right)$ are 


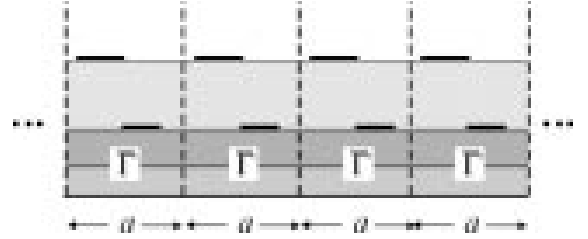

Fig. 2. Laterally periodic line of period $a$.

improper eigenfunctions of the problem, which leads to the following integral FT:

$$
\begin{aligned}
\mathbf{X}(x) & =\frac{1}{2 \pi} \int_{-\infty}^{\infty} \tilde{\mathbf{X}}\left(k_{x}\right) \exp \left(-j k_{x} x\right) d k_{x} \\
\tilde{\mathbf{X}}\left(k_{x}\right) & =\int_{-\infty}^{\infty} \mathbf{X}(x) \exp \left(j k_{x} x\right) d x
\end{aligned}
$$

as the natural spectral representation [16].

After the application of the integral FT, the following matrix firstorder differential equation has to be solved:

$$
\left.\begin{array}{c}
{\left[\mathcal{D}\left(\partial / \partial y, k_{x}, k_{z}, \omega\right)\right][\tilde{\mathbf{X}}]=[\mathbf{Q}(\omega)][\tilde{\mathbf{X}}]} \\
\operatorname{BC} \operatorname{s}(y)
\end{array}\right\} .
$$

Applying the Green's function method along the normal $y$-direction can now solve for the above differential equation.

It is very important to note that in writing (6), it has made use of the fact that the substrate layers are homogoneous along the whole lateral $x$-direction $(-\infty<x<\infty)$, namely, the constitutive dyadics of the problem do not depend on $x$. This fact is relevant when taking the FT of $\mathbf{D}$ - and $\mathbf{B}$-fields. Thus, if

$$
\mathbf{D}(x)=\bar{\epsilon} \cdot \mathbf{E}(x)+\overline{\boldsymbol{\xi}} \cdot \mathbf{H}(x)
$$

then the corresponding spectral counterpart is given by

$$
\tilde{\mathbf{D}}\left(k_{x}\right)=\overline{\boldsymbol{\epsilon}} \cdot \tilde{\mathbf{E}}\left(k_{x}\right)+\overline{\boldsymbol{\xi}} \cdot \tilde{\mathbf{H}}\left(k_{x}\right)
$$

i.e., there exists a simple linear relation between the spectral components of the fields.

\section{B. Laterally Periodic Lines}

Since the structure under study (as that shown in Fig. 2) is fully periodic, a series FT

$$
\mathbf{X}(x)=\frac{1}{a} \sum_{n} \tilde{\mathbf{X}}_{n} \exp \left(-j k_{x, n} x\right), \quad k_{x, n}=n \frac{2 \pi}{a}
$$

can be applied to solve (3). As in the previous case, due to the homogeneity of the layered medium for all the $x$-range, it can be written that

$$
\tilde{\mathbf{D}}_{n}=\overline{\boldsymbol{\epsilon}} \cdot \tilde{\mathbf{E}}_{n}+\overline{\boldsymbol{\xi}} \cdot \tilde{\mathbf{H}}_{n}
$$

and the following ordinary matrix differential equation is reached:

$$
\left.\begin{array}{c}
{\left[\mathcal{D}\left(\partial / \partial y, k_{x, n}, k_{z}, \omega\right)\right]\left[\tilde{\mathbf{X}}_{n}\right]=[\mathbf{Q}(\omega)]\left[\tilde{\mathbf{X}}_{n}\right]} \\
\operatorname{BC} \operatorname{Co}^{\prime}(y)
\end{array}\right\} .
$$

The laterally periodic case is then equivalent to the laterally open case, except that the continuous Fourier variable $\left(k_{x}\right)$ is now replaced by the discrete Fourier variable ( $k_{x, n}$; namely, the harmonics involved in the Fourier expansion of the sources). It should be highlighted that, for the laterally open and periodic cases, the SDA could be efficiently applied because the substrate layers of the structures were homogeneous along the lateral direction.

\section{Laterally Shielded Lines}

If FT techniques are wished to be applied to the shielded structure of Fig. 1, it is first necessary to form an equivalent periodic line (EPL), having exactly the same propagation characteristics. The unit cell or basis-period line (BPL) defining such an EPL is generated by using the well-known technique of images to replace the EWs/MWs by a series of equivalent sources and media. It should be noticed that the imaging of sources implies the proper reflection of all the original sources, namely, both the free/imposed and induced sources (polarization charges, magnetization currents, etc.) by the lateral walls. Since the effect of the induced charges is accounted for by the dyadic constitutive parameters, the imaging of the induced sources will be reflected by the imaged constitutive dyadics. Thus, the whole imaging procedure can be seen as a reflection operation of both the original free/imposed sources and the constitutive dyadics of the media. Although, for the isotropic case, the imaged medium coincides with the original one, this does not happen, in general, for nonisotropic media. (This latter fact is what seems to be obviated by some SDA practitioners.)

A reflection by a plane located at $x=0$ is accounted for by the following linear operator:

$$
\overline{\mathbf{R}}=-\hat{\mathbf{x}} \hat{\mathbf{x}}+\hat{\mathbf{y}} \hat{\mathbf{y}}+\hat{\mathbf{z}} \hat{\mathbf{z}}=\overline{\mathbf{R}}^{-1}
$$

If the symmetry plane is an EW, then $\overline{\mathbf{R}}_{\mathrm{EW}}=-\overline{\mathbf{R}}$ and $\overline{\mathbf{R}}_{\mathrm{MW}}=\overline{\mathbf{R}}$ for an MW. The proper application of the above operator to the original free/imposed sources will give the imaged sources. Obtaining the imaged constitutive dyadics will be illustrated, making use of how field vectors/pseudovectors reflect.

The imaged fields are given by

$$
\begin{aligned}
& \left\{\begin{array}{l}
\mathbf{E}_{R} \\
\mathbf{D}_{R}
\end{array}\right\}=\overline{\mathbf{R}}_{(\mathrm{EW} / \mathrm{MW})} \cdot\left\{\begin{array}{l}
\mathbf{E} \\
\mathbf{D}
\end{array}\right\} \\
& \left\{\begin{array}{l}
\mathbf{H}_{R} \\
\mathbf{B}_{R}
\end{array}\right\}=-\overline{\mathbf{R}}_{(\mathrm{EW} / \mathrm{MW})} \cdot\left\{\begin{array}{l}
\mathbf{H} \\
\mathbf{B}
\end{array}\right\} .
\end{aligned}
$$

Taking into account the general constitutive equations given in (1), after straightforward algebra, the following constitutive relations are found for the reflected fields:

$$
\begin{aligned}
& \mathbf{D}_{R}=\left(\overline{\mathbf{R}} \cdot \overline{\boldsymbol{\epsilon}} \cdot \overline{\mathbf{R}}^{-1}\right) \cdot \mathbf{E}_{R}+\left(-\overline{\mathbf{R}} \cdot \overline{\boldsymbol{\xi}} \cdot \overline{\mathbf{R}}^{-1}\right) \cdot \mathbf{H}_{R} \\
& \mathbf{B}_{R}=\left(\overline{\mathbf{R}} \cdot \overline{\boldsymbol{\mu}} \cdot \overline{\mathbf{R}}^{-1}\right) \cdot \mathbf{H}_{R}+\left(-\overline{\mathbf{R}} \cdot \overline{\boldsymbol{\zeta}} \cdot \overline{\mathbf{R}}^{-1}\right) \cdot \mathbf{E}_{R}
\end{aligned}
$$

which implies that the reflected constitutive dyadics are then given by

$$
\begin{aligned}
& \left\{\begin{array}{l}
\overline{\boldsymbol{\epsilon}}_{R} \\
\overline{\boldsymbol{\mu}}_{R}
\end{array}\right\}=\overline{\mathbf{R}} \cdot\left\{\begin{array}{l}
\overline{\boldsymbol{\epsilon}} \\
\overline{\boldsymbol{\mu}}
\end{array}\right\} \cdot \overline{\mathbf{R}}^{-1} \\
& \left\{\begin{array}{l}
\overline{\boldsymbol{\xi}}_{R} \\
\overline{\boldsymbol{\zeta}}_{R}
\end{array}\right\}=-\overline{\mathbf{R}} \cdot\left\{\begin{array}{l}
\overline{\boldsymbol{\xi}} \\
\bar{\zeta}
\end{array}\right\} \cdot \overline{\mathbf{R}}^{-1} .
\end{aligned}
$$

Carrying out the appropriate reflection operations, Fig. 3 shows the BPLs for three different common situations appearing in practice. In some cases, the reflected dyadics $\left(\overline{\boldsymbol{\Gamma}}_{R}\right.$ in Fig. 3) can coincide with the original ones $(\overline{\boldsymbol{\Gamma}})$, thus, giving place to a BPL that is $x$-homogeneous in its whole period of definition. Unless this latter situation is found, the application of the discrete FT to solve for (3) is not advantageous [13].

In order to clarify the above point, the rectangular waveguide loaded with a longitudinal magnetized ferrite shown in Fig. 4(a) will be considered. Taking into account that, in the present case, the external biasing magnetic field changes its sign under reflection in the conducting plane 


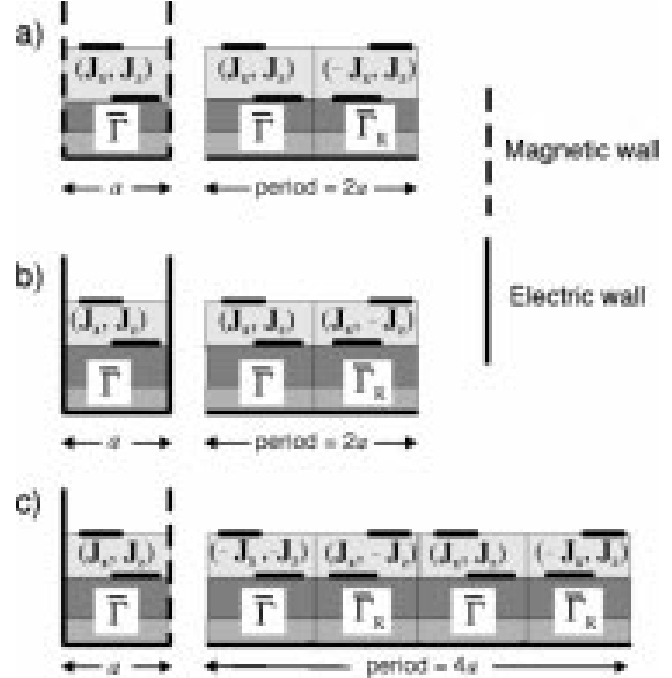

Fig. 3. Original lines (left-hand side) and BPLs of the EPL (right-hand side) resulting from the reflections by the lateral BCs for three different situations. (a) MWs. (b) EWs. (c) E/MWs. $\bar{\Gamma}$ represents any of the constitutive dyadics.

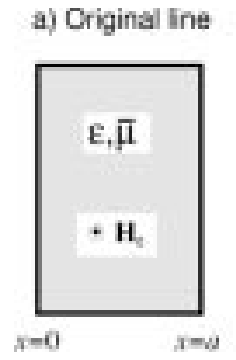

b) Basis-period line

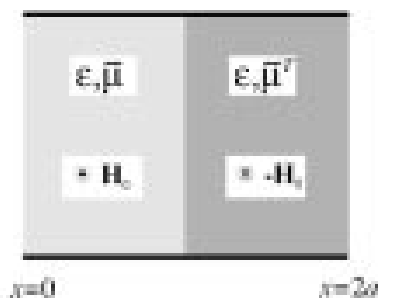

Fig. 4. (a) Shielded rectangular waveguide filled by a longitudinally magnetized ferrite. (b) BPL of the EPL actually analyzed by the SDA.

[12], [17] (which is equivalent to reflect the Polder tensor), the corresponding BPL of the EPL is that shown in Fig. 4(b). Since the dyadic permeability of the BPL is given by

$$
\overline{\boldsymbol{\mu}}^{(\mathrm{BPL})}(x)=\left\{\begin{array}{lc}
\overline{\boldsymbol{\mu}}, & 0<x<a \\
\overline{\boldsymbol{\mu}}^{T}, & a<x<2 a
\end{array}\right.
$$

it is found that the spectral counterpart of

$$
\mathbf{B}(x)=\overline{\boldsymbol{\mu}}^{(\mathrm{BPL})}(x) \cdot \mathbf{H}(x)
$$

is the following convolution product:

$$
\tilde{\mathbf{B}}_{n}=\sum_{m=-\infty}^{\infty} \overline{\boldsymbol{\mu}}_{n-m}^{(\mathrm{BPL})} \cdot \tilde{\mathbf{H}}_{m} .
$$

Thus, all the spectral components of $\mathbf{H}$ are related to all the spectral components of $\mathbf{B}$. This gives rise to an interdependence among all the spectral components of the different fields that would lead to the following system of differential equations:

$$
\left.\begin{array}{c}
{\left[\mathcal{D}\left(\partial / \partial y, k_{x, n}, k_{z}, \omega\right)\right]\left[\tilde{\mathbf{X}}_{n}\right]=\sum_{m=-\infty}^{\infty}\left[\mathbf{Q}_{n-m}(\omega)\right]\left[\tilde{\mathbf{X}}_{m}\right]} \\
\operatorname{BC's}(y)
\end{array}\right\} .
$$

Certainly, the rigorous solution of the above system of coupled differential equations is an impracticable task.

In conclusion, the SDA can always be applied to laterally shielded lines, although it is only suitable for those cases satisfying $\overline{\boldsymbol{\Gamma}}_{R}=\overline{\boldsymbol{\Gamma}}$, namely, when the medium of the BPL is homogeneous along the lateral direction.

\section{CONDITION FOR HOMOGENEITY OF THE BPL}

At the light of (17) and (18), the condition for the existence of the required reflection symmetry $\overline{\boldsymbol{\Gamma}}_{R}=\overline{\boldsymbol{\Gamma}}$ can be expressed as

$$
\begin{aligned}
& \overline{\mathbf{R}} \cdot\left\{\begin{array}{l}
\overline{\boldsymbol{\epsilon}} \\
\overline{\boldsymbol{\mu}}
\end{array}\right\}=\left\{\begin{array}{l}
\overline{\boldsymbol{\epsilon}} \\
\overline{\boldsymbol{\mu}}
\end{array}\right\} \cdot \overline{\mathbf{R}} \\
& \overline{\mathbf{R}} \cdot\left\{\begin{array}{l}
\overline{\boldsymbol{\xi}} \\
\bar{\zeta}
\end{array}\right\}=-\left\{\begin{array}{l}
\bar{\xi} \\
\bar{\zeta}
\end{array}\right\} \cdot \overline{\mathbf{R}} .
\end{aligned}
$$

Thus, the conditions to keep unchanged the medium after the corresponding reflection impose that the constitutive dyadics must have the following generic explicit form:

$$
\begin{aligned}
& \overline{\boldsymbol{\epsilon}}=\epsilon_{x x} \hat{\mathbf{x}} \hat{\mathbf{x}}+\left(\epsilon_{y y} \hat{\mathbf{y}} \hat{\mathbf{y}}+\epsilon_{y z} \hat{\mathbf{y}} \hat{\mathbf{z}}+\epsilon_{z y} \hat{\mathbf{z}} \hat{\mathbf{y}}+\epsilon_{z z} \hat{\mathbf{z}} \hat{\mathbf{z}}\right) \\
& \overline{\boldsymbol{\mu}}=\mu_{x x} \hat{\mathbf{x}} \hat{\mathbf{x}}+\left(\mu_{y y} \hat{\mathbf{y}} \hat{\mathbf{y}}+\mu_{y z} \hat{\mathbf{y}} \hat{\mathbf{z}}+\mu_{z y} \hat{\mathbf{z}} \hat{\mathbf{y}}+\mu_{z z} \hat{\mathbf{z}} \hat{\mathbf{z}}\right) \\
& \overline{\boldsymbol{\xi}}=\xi_{x y} \hat{\mathbf{x}} \hat{\mathbf{y}}+\xi_{x z} \hat{\mathbf{x}} \hat{\mathbf{z}}+\xi_{y x} \hat{\mathbf{y}} \hat{\mathbf{x}}+\xi_{z x} \hat{\mathbf{z}} \hat{\mathbf{x}} \\
& \overline{\boldsymbol{\zeta}}=\zeta_{x y} \hat{\mathbf{x}} \hat{\mathbf{y}}+\zeta_{x z} \hat{\mathbf{x}} \hat{\mathbf{z}}+\zeta_{y x} \hat{\mathbf{y}} \hat{\mathbf{x}}+\zeta_{z x} \hat{\mathbf{z}} \hat{\mathbf{x}} .
\end{aligned}
$$

The above expressions clearly restrict the kind of substrate layers that can be present in those boxed structures to be analyzed by means of the SDA. Specifically, (25) and (26) establish that the SDA will be suitable for the analysis of those lines having dielectric/magnetic substrates whose permittivity/permeability dyadics show a principal axis normal to the lateral boundary walls. This condition will be satisfied, for example, by isotropic materials, uni/biaxial dielectrics having one of the principal directions along the $x$-direction, and plasmons/ferrites biased by a magnetic field directed in the $x$-direction $\left(\mathbf{H}_{0}=H_{0} \hat{\mathbf{x}}\right)$. For the case of bi(ani)isotropic materials $(\bar{\xi} \neq 0, \bar{\zeta} \neq 0),(27)$ and (28) state that the SDA cannot be rigorously applied to boxed structures having, for instance, biisotropic materials and chiroferrites. In fact, (27) and (28) preclude the use of the SDA for the analysis of boxed lines with almost any kind of bianisotropic media.

If the above considerations were ignored and the SDA was used to study boxed structures not fulfilling the requirements given in this paper, the numerical results obtained could be approximately correct provided the box width is large in comparison with the other dimensions of the structure. Actually, numerical series could be considered in such cases as approximations of the Fourier integrals appearing in the analysis of open lines. The results for these closed structures should rather be considered as approximations of the corresponding laterally open structures. If the influence of the lateral shielding could not be neglected, the results obtained with codes not accounting for the theory in this paper are expected to be inaccurate.

\section{CONCLUSION}

This paper has studied the conditions under which FT techniques can be properly applied to study boxed planar lines involving layers of complex linear media. Since the application of the SDA to study the propagation properties of boxed lines is reduced during the analysis of a unit-cell line of an EPL, only the presence of homogeneous substrate layers in the unit-cell line will allow for a suitable application of the technique. The homogeneous nature of the substrate layers of the BPL is determined by the reflection symmetry properties of the media and, thus, the conditions to be satisfied by a general linear bianisotropic material have been studied. Finally, it has been concluded that the SDA can only be properly applied to the analysis of boxed planar 
lines with complex media whose constitutive dyadics are of the type given in (25)-(28). These expressions preclude the use of the standard SDA to rigorously study boxed planar lines with dielectric/magnetic materials having a principal axis not directed along the $x$-direction biisotropic materials as well as almost any type of bianisotropic material.

\section{REFERENCES}

[1] D. Mirshekar-Syahkal, Spectral Domain Method for Microwave Integrated Circuits. Norwood, MA: Artech House, 1990.

[2] C. M. Krowne, "Fourier transformed matrix method of finding propagation characteristics of complex anisotropic layered media," IEEE Trans. Microwave Theory Tech., vol. MTT-32, pp. 1617-1625, Dec. 1984.

[3] G. Plaza, F. Mesa, and M. Horno, "Study of dispersion characteristics of planar chiral lines," IEEE Trans. Microwave Theory Tech., vol. 46, pp. 1150-1157, Aug. 1998.

[4] F. Medina, M. Horno, and H. Baudrand, "Generalized spectral analysis of planar lines on layered media including uniaxial and biaxial dielectric substrates," IEEE Trans. Microwave Theory Tech., vol. 37, pp. 504-510, Mar. 1989.

[5] M. Geshiro and T. Itoh, "Analysis of a coupled slotline on a double-substrate containing a magnetized ferrite," IEEE Trans. Microwave Theory Tech., vol. 40, pp. 765-768, Apr. 1992.

[6] T. Kitazawa, "Nonreciprocity of phase constants, characteristic impedances, and conductor losses in planar transmission lines with layered anisotropic media," IEEE Trans. Microwave Theory Tech., vol. 43, pp. 445-451, Feb. 1995.
[7] C. M. Krowne, A. A. Mostafa, and K. A. Zaki, "Slot and microstrip guiding structure using magnetoplasmas for nonreciprocal millimeter-wave propagation," IEEE Trans. Microwave Theory Tech., vol. 36, pp. 1850-1859, Dec. 1988.

[8] M. Tsutsumi and T. Asahara, "Microstrip lines using yttrium iron garnet film," IEEE Trans. Microwave Theory Tech., vol. 38, pp. 1461-1467, Oct. 1990.

[9] Y. Chen and B. Beker, "Spectral domain analysis of open and shielded slotlines printed on various anisotropic substrates," IEEE Trans. Microwave Theory Tech., vol. 41, pp. 1872-1877, Nov. 1993.

[10] W. Y. Yin and I. Wolff, "Bilateral coplanar waveguides and periodic microstrip lines in bianisotropic superstrate-substrate structures," J. Electromag. Waves Applicat., vol. 13, no. 2, pp. 259-275, Feb. 1999.

[11] A. A. VanTrier, "Some topics in the microwave application of gyrotropic media," IRE Trans. Antennas Propagat., vol. AP-4, pp. 502-507, July 1956.

[12] C. Vassallo, Theorie des Guides d'Ondes Electromagnetiques. Paris, France: Eyrolles, 1985.

[13] H. Cory, "Wave propagation along a closed rectangular chirowaveguide," Microwave Opt. Technol. Lett., vol. 6, no. 14, pp. 797-800, Nov. 1993.

[14] F. Mesa and M. Horno, "Application of the spectral domain method for the study of surface slow-wave in nonreciprocal planar structures with a multilayered gyroelectric substrate," Proc. Inst. Elect. Eng., pt. H, vol. 141, pp. 193-200, June 1993.

[15] D. M. Pozar, Microwave Engineering. Reading, MA: Addison-Wesley, 1990

[16] D. G. Dudley, Mathematical Foundations for Electromagnetic Theory. New York: IEEE Press, 1991.

[17] P. R. McIsaac, "A general reciprocity theorem," IEEE Trans. Microwave Theory Tech., vol. MTT-27, pp. 340-342, Apr. 1979. 